\title{
Instantons and Jumping Lines
}

\author{
Jacques Hurtubise \\ Département de Mathématiques, U.Q.A.M., C.P.8888, Succursale “A”, Montreal, Quebec, \\ Canada H3C 3P 8
}

\begin{abstract}
We study the behaviour under deformation of holomorphic bundles of rank 2 over $\mathbb{P}_{1}(\mathbb{C})$. This is then applied to the description of the moduli space $\tilde{M}_{n}$ of framed SU(2) instantons of charge $n ; \tilde{M}_{n}$ is shown to map to $\mathbb{C}^{n}$, with equidimensional fibers. We use this to provide a stratification of $\tilde{M}_{n}$ and compute the strata explicitly to codimension 4 . This then yields $\pi_{1}\left(\tilde{M}_{n}\right)=\mathbb{Z}_{2}$, and, for the standard moduli space $M_{n}, \pi_{1}\left(M_{n}\right)=0$ for $n$ odd, $\mathbb{Z}_{2}$ for $n$ even.
\end{abstract}

\section{Introduction}

By twistor methods, instantons are known to be equivalent to holomorphic vector bundles on $\mathbb{P}_{3}(\mathbb{C})$ [1]; using the monad construction of Horrocks [12], a description of all solutions was given in [3]. Still, very little is known about the moduli space of solutions; recent work of Donaldson [6] has, however, reduced the problem to classifying certain semi-stable bundles of zero first Chern class on $\mathbb{P}_{2}=\mathbb{P}_{2}(\mathbb{C})$.

It is then natural to try to use this to classify instantons. It turns out that a convenient method for doing this is to restrict the bundle again, to the family of $\mathbb{P}_{1}(\mathbb{C})$ 's in $\mathbb{P}_{2}$ through a fixed point, and to study the behaviour of the bundle as one varies the $\mathbb{P}_{1}$ in the family. The purpose of this article is thus twofold: to examine the behaviour under deformation of holomorphic vector bundles over $\mathbb{P}_{1}=\mathbb{P}_{1}(\mathbb{C})$, and to apply the information gained to the classification of semi-stable vector bundles over $\mathbb{P}_{2}$ ("bundle" is to be taken throughout to mean "holomorphic bundle"; all the results here concern the classification of holomorphic structures).

We have restricted our attention to bundles of rank two, which correspond to the gauge group $\mathrm{SU}(2)$. We obtain a description of the moduli of SU(2) instantons; it complements the monad theoretic work of Barth [5] on stable 2-bundles, but is more geometric in nature; it has the advantage of being concrete enough for us to compute, for example, the fundamental group. 
Consider then a rank two bundle $E$ over $\mathbb{P}_{1}$ with $c_{1}(E)=0$; one has the theorem of Grothendieck [9], which implies that any such $E$ splits as a sum of line bundles: $E \cong \mathcal{O}(k) \oplus \mathcal{O}(-k), k \geqq 0, k$ unique $\left[\mathcal{O}(j)\right.$ is the $j^{\text {th }}$ tensor power of the hyperplane bundle, $\mathcal{O}(-j)$ its dual]. Consider now a bundle $E$ over $\mathbb{P}_{1} \times U, U$ open $\subset \mathbb{C}^{n} ; E$ splits over each $\mathbb{P}_{1} \times\{x\}, x \in U$, as $\mathcal{O}\left(k_{x}\right) \oplus \mathcal{O}\left(-k_{x}\right), k_{x} \geqq 0$. Let $p: \mathbb{P}_{1} \times U \rightarrow \mathbb{P}_{1}$ be the projection, and let $\mathcal{O}(j)$ on $\mathbb{P}_{1} \times U$ denote the lift $p^{*} \mathcal{O}(j)$ of the line bundle on $\mathbb{P}_{1}$; set $E(j)=E \otimes \mathcal{O}(j)$. Then,

$$
h^{0}\left(\mathbb{P}_{1} \times\{x\}, E(-1)\right)=h^{0}\left(\mathbb{P}_{1}, \mathcal{O}\left(k_{x}-1\right) \oplus \mathcal{O}\left(-k_{x}-1\right)\right)=k_{x} .
$$

Semi-continuity of the $h^{0}$ 's $[10$, III -12$]$ then shows that the generic $k_{x}$ is the minimal one; if $E$ is trivial on a $\mathbb{P}_{1}$, then it is trivial over an open set. Assume that this is the case; then, the lines in the family $U$ over which $E \neq \mathcal{O} \oplus \mathcal{O}$ are called the jumping lines of $E$, and in fact constitute a hypersurface in $U$.

The problem, thus, is essentially a bifurcative one: to study how a bundle jumps. It is this question that we attack in Sect. 2; we compute a versal deformation space, define the multiplicity of a jump, and describe the jumping hypersurface.

In Sect. 3 the above is applied to the study of the moduli space $\tilde{M}_{n}$ of framed SU(2) instantons of charge $n$. This space, by the work of Donaldson [6], is equivalent to that of framed $\mathrm{Sl}(2, \mathbb{C})$ bundles $E$ over $\mathbb{P}_{2}$, with $c_{1}(E)=0, c_{2}(E)=n$, which are trivial along a fixed line. (By a result of Grauert-Mülich, they are semistable.) We pick a point on that line, and blow it up, to obtain a surface $\widetilde{\mathbb{P}}_{2}$ ruled by a pencil of lines. $E$ lifted to $\widetilde{\mathbb{P}}_{2}$ has $n$ jumping lines (with multiplicity) in this pencil; we show how $E$ and its $\operatorname{Sl}(2, \mathbb{C})$ framing is completely determined by the choice of these lines, and some extra information "concentrated" at the jumping lines ( 3 parameters per jump.) The key idea is that bundles on $\mathbb{P}_{2}$ correspond to bundles on $\mathbb{P}_{2}$ trivial over the exceptional divisor $C$; this triviality can be viewed as a connection along $C$, and one is led to classifying local equivalence classes of (bundles + connections). This description is concrete enough to give us $\pi_{1}\left(\tilde{M}_{n}\right)=\mathbb{Z}_{2}$; from this, one deduces for the unframed moduli space of instantons $M_{n}$ that $\pi_{1}\left(M_{n}\right)=0$ if $n$ is odd, $\mathbb{Z}_{2}$ if $n$ is even. The section ends with a description of how to extract a monad from our construction, thus linking the two descriptions of instantons.

\section{The Local Geometry of Jumping Lines}

This section is devoted to the local geometry of a rank two bundle $E$ over a family of $\mathbb{P}_{1}$ 's parametrised by $U$ open in $\mathbb{C}^{n}$, i.e., over $\mathbb{P}_{1} \times U$. Our approach throughout will be to study an associated transition matrix for $E$. Let $z$ be a standard affine coordinate on $\mathbb{P}_{1}$, and let $x=\left(x_{1}, \ldots, x_{n}\right)$ be coordinates on $U$, such that $\{x=0\} \in U . E$ is trivial on $\left\{(z, x) \in \mathbb{P}_{1} \times U, z \neq \infty\right\},\left\{(z, x) \in \mathbb{P}_{1} \times U, z \neq 0\right\}$; we consider transition matrices from $\{z \neq \infty\}$ to $\{z \neq 0\}$. As in the introduction, denote by $\mathcal{O}(k)$ the lift of the line bundle $\mathcal{O}(k)$ on $\mathbb{P}_{1}$ to $\mathbb{P}_{1} \times U$; note that $z^{-k}$ is a standard transition function for $\mathcal{O}(k)$ with respect to our covering. Let $E_{x}$ be the restriction of $E$ to $\mathbb{P}_{1} \times\{x\}$; then; 
Proposition 2.1. Let $E_{0} \cong \mathcal{O}(j) \oplus \mathcal{O}(-j), j \geqq 0$; let $k \geqq j$; then $E$ has a transition matrix, in a neighbourhood of $x=0$, of the form:

$$
T_{E}(x, z)=\left(\begin{array}{cc}
z^{k} & p_{E}(x, z) \\
0 & z^{-k}
\end{array}\right),
$$

where $p_{E}(x, z)=\sum_{i=-k+1}^{k-1} p_{i}(x) z^{i}[p(x, z)=0$ if $k=0]$ and $p(0, z)=0$ if $k=j$.

Proof. One chooses an embedding, at $x=0$, of $\mathcal{O}(-k)$ in $E$, i.e., a nowhere zero section of $E(k)$; if $k=j$, choose the natural one. We show that this section extends over a neighbourhood of $x=0$. Write $\mathbb{P}_{1}^{(n)}$ for the $n^{\text {th }}$ formal neighbourhood of $\mathbb{P}_{1} \times\{0\}$ in $U$; one has the exact sequence,

$$
0 \rightarrow E(k) \otimes S^{n}\left(N^{*}\right) \rightarrow E(k) \otimes \mathcal{O}_{\mathbb{P}_{1}}(n) \rightarrow E(k) \otimes \mathcal{O}_{\mathbb{P}_{1}}(n-1) \rightarrow 0,
$$

where $S^{n}\left(N^{*}\right)$ is the $n^{\text {th }}$ symmetric power of the conformal sheaf of $\mathbb{P}_{1} \times\{0\}$ in $\mathbb{P}_{1} \times U$. This bundle is trivial; as $H^{1}\left(\mathbb{P}_{1}, \mathcal{O}(k-j) \oplus \mathcal{O}(k+j)\right)=0$, any section of $E(k)$ on the $(n-1)^{\text {th }}$ formal neighbourhood extends to the $n^{\text {th }}$. By Grothendieck's theorem on formal functions [8], the section extends to an actual neighbourhood $\mathbb{P}_{1} \times V$, and so one has over $\mathbb{P}_{1} \times V: 0 \rightarrow \mathcal{O}(-k) \rightarrow E \rightarrow \mathcal{O}(k) \rightarrow 0$ and, corresponding to this, a transition matrix:

$$
\left(\begin{array}{cc}
z^{k} & p(x, z) \\
0 & z^{-k}
\end{array}\right)
$$

$[p(0, z)=0$ if $j=k]$. Pre-multiplying by matrices holomorphic in $x, z^{-1}$, and postmultiplying by matrices holomorphic in $x, z$ (i.e. changing trivialisations) one sees that one may kill in $p$ all terms of order $\leqq-k$ or $\geqq k$ in $z$.

Corollary 2.3. $\mathbb{C}^{2 k-1}$ is a minimal local versal deformation space for a bundle $E \cong \mathcal{O}(k) \oplus \mathcal{O}(-k)$ over $\mathbb{P}_{1}$.

Proof. Let $s_{i}, i=-k+1, \ldots, k-1$ be coordinates on $\mathbb{C}^{2 k-1}$; define a transition matrix for a bundle $F$ over $\mathbb{P}_{1} \times \mathbb{C}^{2 k-1}$ as the $T_{F}$ associated to $p_{F}(s, z)=\sum s_{i} z^{i}$. Then, for any bundle $E$ over $\mathbb{P}_{1} \times U$, if $p_{i}(x)$ are the functions obtained for $E$ in Proposition 2.1, map $U$ to $\mathbb{C}^{2 k-1}$ by $x \rightarrow\left(p_{i}(x)\right)$.

If $p$ is the corresponding map $\mathbb{P}_{1} \times U \rightarrow \mathbb{P}_{1} \times \mathbb{C}^{2 k-1}$, it is clear that $E \cong p^{*} F$.

To see that the dimension $(2 k-1)$ is minimal, we consider one-parameter "linear" deformations given by $p_{E}(x, z)=x \sum p_{i} z^{i}, p_{\tilde{E}}(x, z)=x \sum \tilde{p}_{i} z^{i}$ with at least one $p_{i} \neq 0$. Then one has $E \cong \tilde{E}$ iff $\exists c \neq 0$ with $\left(p_{-k+1}, \ldots, p_{k-1}\right)=c\left(\tilde{p}_{-k+1}, \ldots, \tilde{p}_{k-1}\right)$. For suppose that $E \cong \widetilde{E}$; one then has a nondegenerate section of $E^{*} \otimes \widetilde{E}$. Using the forms (2.2) of the matrices for $E, \widetilde{E}$, one has the matrix for $E^{*} \otimes \widetilde{E}$ :

$$
\left(\begin{array}{cccc}
z^{2 k} & -p_{E} z^{k} & p_{\tilde{E}} z^{k} & -p_{E} p_{\tilde{E}} \\
0 & 1 & 0 & p_{\tilde{E}} z^{-k} \\
0 & 0 & 1 & -p_{E} z^{-k} \\
0 & 0 & 0 & z^{-2 k}
\end{array}\right)
$$

over $x=0$, as $p_{E}=p_{\tilde{E}}=0$, our non-degenerate section over $z \neq \infty$ is of the form $(0, a, b, f(z))^{T}, f(z)$ polynomial of degree $\leqq 2 k, a \neq 0 \neq b, a, b \in \mathbb{C}$. The obstruction 
to the extension of this section to the first formal neighbourhood of $x=0$ lies in $H^{1}\left(\mathbb{P}_{1} \times\{0\}, E^{*} \otimes \tilde{E}\right) \cong H^{1}\left(\mathbb{P}_{1}, \mathcal{O}(-2 k)\right)$ and is precisely $z^{-k}\left(a p_{E}-b p_{\tilde{E}}\right)$ in a standard representation of the cocycle. As the section does extend, $a p_{E}=b p_{\tilde{E}}$. Conversely, if $p_{E}=c p_{\tilde{E}}$, then $(0,1, c, 0)^{T}$ provides a non-degenerate section of $E^{*} \otimes \tilde{E}$.

We now turn to the jumping behaviour of an $E$ with matrix (2.2), but with $p_{E}(0, z)$ not necessarily zero.

Proposition 2.4. Let $p_{E}(x, z)=\sum_{i=k+1}^{k-1} p_{i}(x) z^{i}$. Consider the matrix

$$
\Gamma_{E}(x)=\left(\begin{array}{ccccc}
p_{0}(x), & p_{-1}(x) & \ldots & p_{-k+2}(x), & p_{-k+1}(x) \\
p_{1}(x), & p_{0}(x) & \ldots & p_{-k+3}(x), & p_{-k+2}(x) \\
\vdots & \vdots & & \vdots & \vdots \\
p_{k-2}(x), & p_{k-3}(x) & \ldots & p_{0}(x), & p_{-1}(x) \\
p_{k-1}(x), & p_{k-2}(x) & \ldots & p_{1}(x), & p_{0}(x)
\end{array}\right)
$$

Then $E_{x}$ is of type $(j,-j)$ iff $\Gamma_{E}(x)$ has corank $j$.

Proof. $E_{x}$ is of type $(j,-j)$ iff $E_{x}(-1)$ has $j$ independent sections. A section of $E_{x}(-1)$ is a pair $f(z)=\left(f_{1}(z), f_{2}(z)\right)^{T}$, holomorphic in $z$, such that $z T_{E}(x, z) \cdot f(z)$ is holomorphic in $z^{-1}$ : this means that $\left[z^{k+1} f_{1}(z)+z p(x, z) f_{2}(z)\right]$ and $z^{-k+1} f_{2}(z)$ are holomorphic in $z^{-1}$.

The second condition forces $f_{2}(z)$ to be a polynomial of degree $\leqq(k-1)$; the first then demands that $p_{E}(x, z) \cdot f_{2}(z)$ have no terms of orders $0, \ldots, k-1$ in $z$. Writing the coefficients of $f_{2}$ as a column vector $F$, this is precisely $\Gamma_{E}(x) \cdot F=0$, and the result follows.

For an $E$ with the generic $E_{x}$ of type $(0,0)$, the jumping lines are given by the vanishing of a determinant and so form a hypersurface. Our next task is to see that this vanishing defines the hypersurface with the same multiplicity as that given by the coordinate free definition of Barth ([4], see also [13, pp. 214-220]). If $\Pi: \mathbb{P}_{1} \times U \rightarrow U$ is the projection, the hypersurface is defined there via the intermediary of the direct image sheaf $R^{1} \Pi_{*} E(-1)$, as follows. One chooses a resolution

$$
0 \rightarrow K \rightarrow \oplus \mathcal{O}\left(k_{i}\right) \rightarrow E(-1) \rightarrow 0
$$

with $k_{i}<0$; projecting, one obtains.

$$
0 \rightarrow R^{1} \Pi_{*} K \stackrel{h}{\longrightarrow} R^{1} \Pi_{*}\left(\oplus \mathcal{O}\left(k_{i}\right)\right) \rightarrow R^{1} \Pi_{*} E(-1) \rightarrow 0 .
$$

The first two sheaves are locally free, of the same rank. The hypersurface is then defined by $\operatorname{det}(h)=0$. One shows that this hypersurface has a multiplicity which is independent of the choice of resolution.

We now build a particularly nice resolution of the form (2.5), for an $E$ given by (2.2).

Lemma 2.6. $E$ has a resolution:

$$
0 \rightarrow \mathcal{O}(-k-1)^{\oplus 2 k} \rightarrow \mathcal{O}(-k)^{\oplus 2 k+2} \rightarrow E \rightarrow 0 .
$$


Proof. Maps $\mathcal{O}(-k) \rightarrow E$ are the same as sections of $E(k)$. Referring to the trivialisations corresponding to (2.2) we write out, over the $z \neq \infty$ trivialisation, $2 k+2$ maps from line bundles $V_{0}, V_{1}, W_{1}, \ldots, W_{2 k}$ all isomorphic to $\mathcal{O}(-k)$, to $E$ :

$$
\begin{gathered}
V_{0} \quad V_{1} \quad W_{1} \quad W_{2} \\
\left(\begin{array}{l}
1 \\
0
\end{array}\right)\left(\begin{array}{l}
0 \\
1
\end{array}\right)\left(\begin{array}{l}
0 \\
z
\end{array}\right)\left(\begin{array}{c}
-p_{k-1} z \\
z^{2}
\end{array}\right)\left(\begin{array}{c}
-p_{k-1} z^{2}-p_{k-2} z \\
z^{3}
\end{array}\right)^{\cdots} \\
\cdots\left(\begin{array}{c}
-p_{k-1} z^{2 k-1}-p_{k-2} z^{2 k-2} \ldots-p_{k+1} z \\
z^{2 k}
\end{array}\right.
\end{gathered}
$$

Similarly, maps $\mathcal{O}(-k-1) \rightarrow \mathcal{O}(-k)$ are of the form $(a z+b)$ in the standard trivialisations; thus, setting $U_{1}, \ldots, U_{2 k}$ to be line bundles, all isomorphic to $\mathcal{O}(-k-1)$, one defines the map $\oplus U_{i} \rightarrow V_{0} \oplus V_{1} \oplus\left(\oplus\left(W_{i}\right)\right)$ :

\begin{tabular}{c|cccccccc} 
& $V_{0}$ & $V_{1}$ & $W_{1}$ & $W_{2}$ & $W_{3}$ & $\ldots$ & $W_{2 k-1}$ & $W_{2 k}$ \\
\hline$U_{1}$ & 0 & $-z$ & 1 & 0 & 0 & $\ldots$ & 0 & 0 \\
$U_{2}$ & $p_{k-1} z$ & 0 & $-z$ & 1 & 0 & $\ldots$ & 0 & 0 \\
$U_{3}$ & $p_{k-2^{2}} z$ & 0 & 0 & $-z$ & 1 & $\ldots$ & 0 & 0 \\
$\vdots$ & $\vdots$ & $\vdots$ & $\vdots$ & $\vdots$ & $\vdots$ & $\vdots$ & $\vdots$ & $\vdots$ \\
$U_{2 k-1}$ & $p_{-k+2} z$ & 0 & 0 & 0 & 0 & $\ldots$ & 1 & 0 \\
$U_{2 k}$ & $p_{-k+1} z$ & 0 & 0 & 0 & 0 & $\ldots$ & $-z$ & 1
\end{tabular}

combining, one checks that the composition

$$
0 \rightarrow \oplus U_{i} \rightarrow V_{0} \oplus V_{1} \oplus\left(\oplus W_{i}\right) \rightarrow E \rightarrow 0
$$

is exact.

Proposition 2.7. The two definitions of hypersurface of jumping lines coincide.

Proof. We use the resolution of (2.6), twisted by $\mathcal{O}(-1)$. One must consider the maps induced on cohomology groups by a map $\mathcal{O}(-k-2) \rightarrow \mathcal{O}(-k-1)$ given in standard trivialisations by $s \rightarrow(a z+b) s$. Note that $H^{1}\left(\mathbb{P}_{1}, \mathcal{O}(-k-2)\right)$ has a standard basis of Cech cocycles: $\left(z^{-1}, z^{-2}, \ldots, z^{-k-1}\right) ;$ similarly, for $H^{1}\left(\mathbb{P}_{1}, \mathcal{O}(-k-1)\right):\left(z^{-1}, z^{-2}, \ldots, z^{-k}\right)$. With respect to these bases, the induced map on cohomology $H^{1}\left(\mathbb{P}_{1}, \mathcal{O}(-k-2)\right) \rightarrow H^{1}\left(\mathbb{P}_{1}, \mathcal{O}(-k-1)\right)$ has a $k \times(k+1)$ matrix $a(0, \mathrm{Id})+b(\mathrm{Id}, 0)$. Referring now to the explicit maps in (2.6), one obtains for the map $h$ induced by our resolution a $(2 k+2) k \times 2 k(k+1)$ matrix made up of $k \times(k+1)$ blocks; a tedious but straightforward reduction brings this matrix to a block diagonal form $\operatorname{diag}\left(\Gamma_{E}, \mathrm{Id}\right)$, which proves the result.

The equation $\operatorname{det}\left(\Gamma_{E}\right)=0$ then defines a cone $C$ of degree $k$ in the versal deformation space of $\mathcal{O}(k) \oplus \mathcal{O}(-k)$; one can use this to see the different ways of obtaining a jumping line of multiplicity $k$ in, say, a one-parameter family: either by a curve through the origin, transversal to $C$, or by a line not passing through the origin, of the appropriate degree of tangency to $C$. (The multiplicity of a jump being determined by its embedding in the versal space.) The first case gives a jump of type $(k,-k)$; the latter, one type $(j,-j), j<k$. We now show that, in an 
appropriate sense, jumps of type $(1,-1)$ are dense amongst those of multiplicity $k$, for one dimensional deformations.

Proposition 2.8. Let $V_{k}^{n} \cong \mathbb{C}^{(2 k-1) n}$ be the space of maps of the $n^{\text {th }}$ formal neighbourhood of the origin in $\mathbb{C}$ into the versal deformation space $V_{k}$ of $\mathcal{O}(k) \oplus \mathcal{O}(-k)$ (n-jets). Let $Z^{n} \subset V_{k}^{n}$ be the subvariety of those jets which define $a$ jumping line of multiplicity $n$ at $(x=0)$ in $C$; then the subvariety $U$ of $Z^{n}$ of those jets whose evaluation at $x=0$ corresponds to a jump of type $(1,-1)$ is dense in $Z^{n}$.

Proof. One considers $k \geqq 2$, the case $k=1$ being trivial. For a jet $J$ in $V_{k}^{n}$, let $p_{J}(x, z)$ correspond to the associated polynomial as in (2.2), and let $\Gamma_{J}(x)$ be the associated matrix, as in (2.4); write $\Gamma_{J}(x)=\Gamma_{0}+x \Gamma_{1}+\ldots+x^{n} \Gamma_{n}$. The multiplicity of $J$ is $n$ iff $\operatorname{det}\left(\Gamma_{J}\right)=0$ to order $n$ ( $n$ equations); $J$ corresponds to a jump of type $(j,-j)$ iff corank $\left(\Gamma_{0}\right)=j$. These conditions are projectively invariant, and so one considers $\mathbb{P}\left(Z^{n}\right)$ in $\mathbb{P}\left(V_{k}^{n}\right)$. The set $\mathbb{P}(U)$ of jumps of type $(1,-1)$ is open in $\mathbb{P}\left(Z^{n}\right)$; furthermore it is non-empty [consider $p_{J}(x, z)=z+x^{n} z^{-k+1}$ ]. Therefore the result follows if $\mathbb{P}\left(Z^{n}\right)$ is connected; but this follows immediately, as $\operatorname{codim}\left(\mathbb{P}\left(Z^{n}\right)\right)$ is $n$ in $\mathbb{P}\left(\mathbb{C}^{(2 k-1) n}\right)$, and varieties in $\mathbb{P}^{m}$ of dimension greater than $m / 2$ are connected.

Remark. As vanishing to order $n$ depends solely on the $n$-jets, a similar statement about density of jumps of type $(1,-1)$ can be made for germs of maps $\mathbb{C} \rightarrow V_{k}$.

We saw in Corollary (2.4) that the dimension of the versal deformation space $V_{k}$ was determined simply by considering "linear" deformations $E$ given by $p_{E}(x, z)$ $=x \sum p_{i} z^{i}$. We now show that any such one parameter deformation can be given such a linear form, provided that it is transversal to the cone $C$ of jumping lines in the versal deformation space.

Proposition 2.9. Let $E, E$ be two one parameter deformations of $\mathcal{O}(k) \oplus \mathcal{O}(-k)$, corresponding, as in (2.2) to Laurent polynomials (in $z$ ) $p_{\tilde{E}}(x, z)=x \tilde{p}_{1}(z), p_{E}(x, z)$ $=x p_{1}(z)+x^{2} p_{2}(z)+\ldots$, with $\quad \tilde{p}_{1}(z)=p_{1}(z)$. Write $\Gamma_{\tilde{E}}(x)=x \tilde{\Gamma}_{1}, \quad \Gamma_{E}(x)=x \Gamma_{1}$ $+x^{2} \Gamma_{2}+\ldots$ and suppose that $\operatorname{det}\left(\tilde{\Gamma}_{1}\right)=\operatorname{det}\left(\Gamma_{1}\right) \neq 0$. Then $E$ is isomorphic to $\widetilde{E}$ near $x=0$.

Proof. The idea is to trivialise $E, \widetilde{E}$ over $x \neq 0$ by sections; using these, to identify $E$ and $\tilde{E}$ in a natural way; and then to check that this identification extends smoothly to $x=0$.

We first compute sections of $E$. Write a section of $E$, over $z \neq \infty$, with respect to the trivialisation corresponding to $(2.2)$, as $(\alpha(x, z), \beta(x, z))^{T}$. One can write

$$
\begin{gathered}
\alpha(x, z)=\sum \alpha_{i j} x^{i} z^{j}=\alpha_{1}(z) x+\alpha_{2}(z) x^{2}+\ldots, \\
\beta(x, z)=\sum \beta_{i j} x^{i} z^{j}=\beta_{0}(z)+\beta_{1}(z) x+\ldots
\end{gathered}
$$

From the condition $T_{E}(x, z) \cdot(\alpha(x, z), \beta(x, z))^{T}=\left(\right.$ holom. in $\left.x, z^{-1}\right)$, one deduces that $\beta_{i}, \alpha_{i}$ are polynomial in $z, \beta_{i}$ of degree $\leqq k, \alpha_{i}$ of degree $\leqq k-1$. One then computes $\alpha, \beta$ order by order in $x$. For example, on the first formal neighbourhood of $x=0$ one obtains:

$$
\Gamma_{1} \cdot\left(\beta_{0,1}, \ldots, \beta_{0, k}\right)^{T}=\left(-\beta_{0,0} p_{1,1},-\beta_{0,0} p_{1,2}, \ldots,-\beta_{0,0} p_{1, k-1},-\alpha_{1,0}\right)^{T}
$$

and

$$
M_{E} \cdot\left(\beta_{01}, \ldots, \beta_{0 k}\right)^{T}=\left(-\alpha_{1,1}, \ldots,-\alpha_{1, k-1}\right)^{T},
$$


where $\left(M_{E}\right)_{i, j}=p_{1, k-1+i-j}$, if $i \geqq j$ and zero if $i<j$. Similarly, the $j^{\text {th }}$ order neighbourhood yields

$$
\begin{gathered}
\Gamma_{1} \cdot\left(\beta_{j-1,1}, \ldots, \beta_{j-1, k}\right)^{T}=\left(-\beta_{j-1,0} p_{1,1},-\beta_{j-1,0} p_{1,2}, \ldots,-\beta_{j-1,0} p_{1, k-1},-\alpha_{j, 0}\right)^{T} \\
+f(\text { lower order terms (in } x) \text { of } \beta, \alpha) . \\
M_{E}\left(\beta_{j-1,1}, \ldots, \beta_{j-1, k}\right)^{T}= \\
\left(-\alpha_{j, 1}, \ldots, \alpha_{j, k}\right) \\
+g \text { (lower order terms (in } x) \text { of } \beta, \alpha) .
\end{gathered}
$$

One notes that:

i) As $\Gamma_{1}$ is invertible, fixing arbitrarily all $\alpha_{i, 0}$, for $i \geqq 1, \beta_{i, 0}$, for $i \geqq 0$ then gives the other terms $\left(\alpha_{0,0}\right.$ is zero).

ii) Restriction to the $j^{\text {th }}$ order neighbourhood determines $\alpha_{i}(z) \forall i \leqq j$, and $\beta_{i}(z) \forall i \leqq j-1$.

There are therefore two unique sections $s^{1}=\left(\alpha^{1}, \beta^{1}\right), s^{2}=\left(\alpha^{2}, \beta^{2}\right)$ such that

$$
N(x, 0)=\left(\begin{array}{ll}
\alpha^{1}(x, 0), & \alpha^{2}(x, 0) \\
\beta^{1}(x, 0), & \beta^{2}(x, 0)
\end{array}\right)=\left(\begin{array}{ll}
x, & 0 \\
0, & 1
\end{array}\right) .
$$

One then has, for $N(x, z)$ :

$$
N(x, z)=\left(\begin{array}{cc}
0, & 0 \\
\beta_{0}^{1}(z), & \beta_{0}^{2}(z)
\end{array}\right)+x\left(\begin{array}{cc}
\alpha_{1}^{1}(z), & \alpha_{1}^{2}(z) \\
\beta_{1}^{1}(z), & \beta_{1}^{2}(z)
\end{array}\right)+x^{2} \ldots .
$$

(One checks that convergence poses no problem.) Now do the same with $\tilde{E}$, obtaining $\tilde{s}^{1}, \tilde{s}^{2}, \tilde{N}$ : one can identify $E$ with $\tilde{E}$ over $x \neq 0$ by identifying $s^{i}$ with $\tilde{s}^{i}$. This, in terms of our trivialisations, is just $N^{-1} \tilde{N}$; therefore one wants $N^{-1} \tilde{N}$ to be holomorphic at $x=0$. Note now that the matrix $T_{E}(x, z)$ induces the transition function 1 for $\Lambda^{2} E$; as $\operatorname{det}(N(x, z))$ represents a section of $\Lambda^{2}(E)$ in such a trivialisation, $\operatorname{det}(N(x, z))=\operatorname{det}(N(x, 0))=x$. Using this, the fact that $p_{1}(z)=\tilde{p}_{1}(z)$ and remark ii) above, (which implies that $\alpha_{1}^{i}=\tilde{\alpha}_{1}^{i}, \beta_{0}^{i}=\widetilde{\beta}_{0}^{i}$ ) one computes that $N^{-1} \tilde{N}=x^{-1} N^{\text {adj }} \tilde{N}$ is indeed holomorphic, and that $\operatorname{det}\left(N^{-1} \tilde{N}\right)=1$.

Remark 2.15. By the same type of method, one can show that a one parameter deformation $E$ of $\mathcal{O}(1) \oplus \mathcal{O}(-1)$, of multiplicity $k$, can be given $p_{E}(x, z)=x^{k}$.

Remark. The above suggests the generalisation that the bundle corresponding to a curve in $V_{k}$ is determined by its intersection with the first formal neighbourhood $C^{1}$ of the cone $C$ in $V_{k}$.

\section{A Description of Instanton Moduli}

\section{A) Preliminary Remarks}

The objects of study in this section will be semi-stable rank two bundles $E$ over $\mathbb{P}_{2}$ with $c_{1}(E)=0, c_{2}(E)=n$. Recall that a locally free sheaf $F$ of rank $r$ over $\mathbb{P}_{m}$ is $\{$ semi $\}$ stable iff for all coherent subsheaves $F^{\prime}$ of rank $s, 0<s<r$,

$$
\left(c_{1}\left(F^{\prime}\right) / s_{\{\leqq\}}^{<}\left(c_{1}(F) / r\right)\right.
$$


if rank $E=2, E$ can be tensored by a suitable $\mathcal{O}(j)$ so that $c_{1}(E)=0$ or -1 ; then, $E$ is stable iff $H^{0}\left(\mathbb{P}_{m}, E\right)=0$, and, if $c_{1}(E)=0, E$ is semi-stable iff $H^{0}\left(\mathbb{P}_{m}, E(-1)\right)=0$.

A natural operation on a bundle over $\mathbb{P}_{m}$ is restriction to a line:

Theorem 3.1 (Grauert-Mülich [7]). A rank two bundle E, with $c_{1}(E)=0$, over $\mathbb{P}_{m}$ is semi-stable iff there is a line over which it is trivial (iff it is trivial when restricted to the generic line).

The set of lines over which $E$ is non-trivial is, as we have seen, a hypersurface; in particular, one has:

Proposition 3.2 (Barth [4]). The set of lines over which a rank two semi-stable bundle $E$ over $\mathbb{P}_{2}$ with $c_{1}(E)=0, c_{2}(E)=n$ is non-trivial is a curve $J$ in $\mathbb{P}_{2}^{*}$ of degree $n$.

Barth also shows that if the jumping type of a line is $(j,-j)$, and its multiplicity is $k$, then $j \leqq k$; alternatively, this can be deduced here from the results of Sect. 2. In particular, if $J$ is reduced, then the generic jump is of type $(1,-1)$.

Finally, the link with instantons is given by the following theorem of Donaldson, which establishes an equivalence of framed moduli spaces; it is given here for the case of $\mathrm{SU}(2)$.

Theorem 3.3 [6]. Let $\tilde{M}(\mathrm{SU}(2), n)$ be the manifold of isomorphism classes of pairs (SU(2) instanton bundle $F$ on $\mathbb{P}_{3}$ of charge $n ; S U(2)$ frame on $F$ over a real line $l_{\infty}$ in $\mathbb{P}_{3}$ ). Fix a $\mathbb{P}_{2}$ in $\mathbb{P}_{3}$ containing $l_{\infty}$; let $O \tilde{M}(\operatorname{Sl}(2, \mathbb{C}), n)=\tilde{M}_{n}$ be the moduli space of pairs (holomorphic rank 2 bundle $E$ on $\mathbb{P}_{2}$ with $c_{1}(E)=0, c_{2}(E)=n$, trivial over $l_{\infty}$; trivialisation of $E$ over $\left.l_{\infty}\right)$. Then there is a natural isomorphism

$$
\tilde{M}(\mathrm{SU}(2), n) \stackrel{R}{\longrightarrow} O \tilde{M}(\mathrm{Sl}(2, \mathbb{C}), n)
$$

given simply by restriction.

The advantage of this isomorphism is that all considerations of reality disappear from the description of instantons, leaving one only with the task of classifying complex holomorphic objects.

\section{B) The Moduli Space}

i) Consider then a 2-bundle $E$ on $\mathbb{P}_{2}$ trivial over $l_{\infty}$; pick once and for all a point $p$ of $l_{\infty}$, and blow up $\mathbb{P}_{2}$ at $p$ to obtain $\widetilde{\mathbb{P}}_{2}$, with a projection $f: \widetilde{\mathbb{P}}_{2} \rightarrow \mathbb{P}_{2}$. The pencil of lines through $p$ then lifts to a ruling of $\widetilde{\mathbb{P}}_{2}$; the lines in the ruling are parametrised by their point of intersection with $C=f^{-1}(p)$, the exceptional divisor. Lifting a bundle $E$ on $\mathbb{P}_{2}$, one obtains a bundle $\tilde{E}$ on $\widetilde{\mathbb{P}}_{2}$, trivial over $C$; conversely, starting with such an $\tilde{E}$, one can invert the process (see, e.g. Atiyah [2]) and push down to obtain a bundle $E$. As $C=\mathbb{P}_{1}$ is one dimensional and simply connected, one can "localise" the triviality of $\tilde{E}$ over $C$ by making the equivalent statement that $E$ has a holomorphic connection along $C$. In a similar fashion, consider a choice of trivialisation of $E$ over $l_{\infty}$; this is equivalent to a choice of trivialisation over $p$. Lifting to $\tilde{E}$, this becomes a trivialisation along $C$, which simultaneously determines the connection by being a basis of flat sections. Thus, one has: 
Proposition 3.4. There is an equivalence:

$\begin{array}{lll}\text { (bundles over } \mathbb{P}_{2} \text {, trivial } & \stackrel{f^{*}}{\longrightarrow} \begin{array}{l}\text { (bundles over } \mathbb{P}_{2} \text {, trivial over } \\ \text { over } l_{\infty} \text { with a fixed } \\ \text { trivialisation there) }\end{array} \longleftarrow \tilde{l}_{\infty} \cup C \text { with a fixed trivialisation } \\ \text { ("framing") along } C \text { ). }\end{array}$

Here $\tilde{l}_{\infty}$ is the proper transform of $l_{\infty}$. We shall call bundles on $\mathbb{P}_{2}$ equipped with a choice of trivialisation along $C$ framed bundles.

The "local" nature of the trivialisation along $C$ suggests that one should first look at open sets $U \subsetneq C$; the union of the lines of the ruling through $U$ is an open set $V \cong \mathbb{P}_{1} \times U$ of $\widetilde{\mathbb{P}}_{2}$. Let $E, E^{\prime}$ be two framed bundles over $\mathbb{P}_{2}$; suppose that they are both trivial along the lines of the ruling in $V$. Then, there is a unique isomorphism $E \cong E^{\prime}$ preserving the framing: one maps the fixed trivialisations along $U$ to each other, and, as the bundles are trivial on the lines of the ruling, this map extends canonically to all of $V$.

For rank two bundles, (3.2) tells us that there are $n$ jumping lines, counting multiplicity, in the ruling ("in $C$ "). Suppose that $E, E^{\prime}$ have the same jumping lines in $C$; then, if the canonical isomorphism defined away from the jumping lines were to extend to all of $\widetilde{\mathbb{P}}_{2}$, one would have an equivalence of framed bundles $E \cong E^{\prime}$. The problem of moduli is then "localised" around the jumping lines; one must classify equivalence classes of framed one parameter deformations of jumping lines. More precisely, define a (rank two) one parameter jump to be a 2-bundle $E$ over $\mathbb{P}_{1} \times U$, open in $\mathbb{C}, 0 \in U$, with $E$ trivial over $\mathbb{P}_{1} \times\{x\}, x \neq 0$ and $E$ not trivial over $\mathbb{P}_{1} \times\{0\}$. The multiplicity of such a jump was defined in Sect. 2 . Now pick a point $q \in \mathbb{P}_{1}$; a framing of the one parameter jump is the choice of a trivialisation of $E$ along $\{q\} \times U$. Two framed jumps will be equivalent iff they are isomorphic by a map sensing the framing of one into the other. Then:

Theorem 3.5. Let $\infty$ be the point in $C$ corresponding to $l_{\infty}$ in $\mathbb{P}_{2}$. There is a natural map:

$$
\prod: \tilde{M}_{n} \rightarrow S^{n}(C \backslash\{\infty\})=\mathbb{C}^{n} .
$$

from $\tilde{M}_{n}$ into the $n^{\text {th }}$ symmetric power of $C \backslash\{\infty\}$, whose fiber at a point $\sum_{i=1}^{r} m_{i} p_{i}$, $p_{i} \in C \backslash\{\infty\}, m_{i} \in \mathbb{N}, \sum_{i=1}^{r} m_{i}=n$ is the product

$$
Q_{m_{1}} \times Q_{m_{2}} \times \ldots \times Q_{m_{r}},
$$

where $Q_{k}$ is the space of equivalence classes of germs of framed one parameter jumps of multiplicity $k$. All of the fibers of $\Pi$ are of complex dimension $3 n$.

Proof. The map $\prod$ just associates to $E$ its divisor of jumping lines in the ruling. As noted above, if two framed bundles $E$ and $E^{\prime}$ are isomorphic at all their jumping lines in $C$ via the mapping derived from the framing, then they are isomorphic; the framed jumps at $p_{i}$ determine the bundle.

Conversely, suppose given any germs $E_{i}, i=1, \ldots, r$, of framed one parameter jumps of multiplicity $m_{i}$, defined over open sets $\mathbb{P}_{1} \times U_{i}, p_{i} \in U_{i}$ and which jump at $p_{i}$; taking in addition a trivial framed bundle $E_{0}$ over

$$
\mathbb{P}_{2} \backslash\{\text { union of the jumping lines }\} \cong \mathbb{P}_{1} \times U_{0}, U_{0}=C \backslash\left\{p_{1}, \ldots, p_{r}\right\},
$$


one can glue together $E_{i}$ and $E_{j}$ in a canonical way over $P_{1} \times\left(U_{i} \cap U_{j}\right)$ by using the framing; one obtains a globally defined $E$. The fibers of $\Pi$ are thus as described; their equidimensionality will be proven once we have given a discription of the $Q_{i}$.

ii) The Structure of $Q_{1}$. As we have already remarked, a jump of multiplicity one must be of type $(1,-1)$; referring to (2.2), (2.9) such a 2-bundle $E$ can be given a transition matrix, for the standard covering of $\mathbb{P}_{1} \times U$, of the form:

$$
\left(\begin{array}{cc}
z & x \\
0 & z^{-1}
\end{array}\right)
$$

where $z$ is an affine coordinate on $\mathbb{P}_{1}, x$ a coordinate on $U$; thus all our multiplicity one jumps are holomorphically equivalent (without framings). Now suppose that the point $q$ for the framing is $(z=0)$. The space of all possible framings is obtained from the canonical one corresponding to the trivialisation over $z \neq \infty$ implicit in (3.6), by the action of the group $\mathrm{Gl}(2, \mathbb{C})_{U}$ of holomorphic maps $U \rightarrow \mathrm{Gl}(2, \mathbb{C})$. Note that each such map induces a bundle isomorphism over the non-jumping lines; the space $Q_{1}$ will be the quotient of $\mathrm{Gl}(2, \mathbb{C})_{U}$ by the subgroup $H_{U}$ of those maps which induce holomorphic isomorphisms even at the jump. One way to express the property that $g(x)$ belongs to $H_{U}$ is as follows: away from $x=0$, one has sections of $E$ given over $z \neq \infty$ by $(1,-z / x)^{T},(0,1)^{T}$ and over $z \neq 0$ by $(0,-1)^{T},(x, 1 / z)^{T}$. Using these, define a trivialisation by sections, away from $x=0$. One can form the diagram of transition matrices: (set $W=\{z \neq 0\}, V=\{z \neq \infty\}$ )

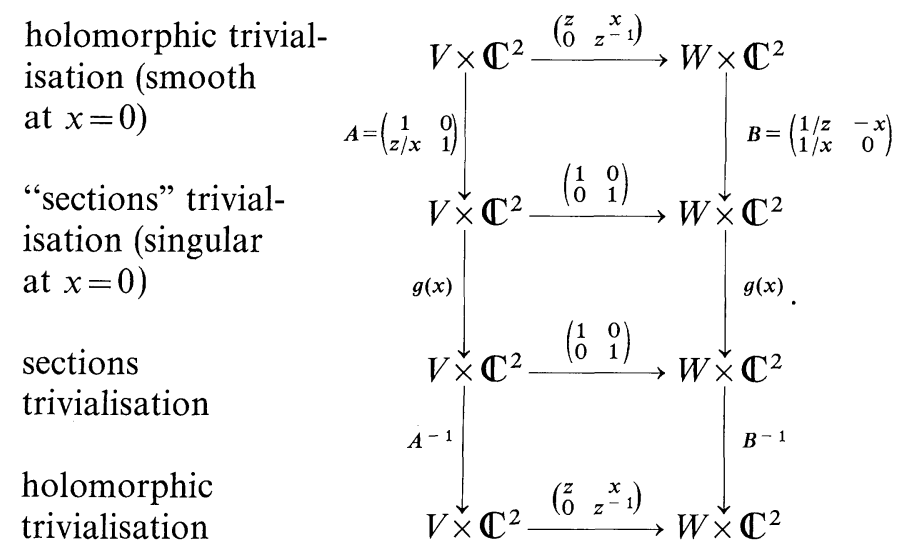

The condition that $g(x) \in H_{U}$ is then that $A^{-1} g A, B^{-1} g B$ be holomorphic, nonsingular at $x=0$. Writing $g(x)=\left(g_{i j}(x)\right) i, j=1,2$, one finds that the conditions are:

$$
\left(g_{12}(x)\right) / x^{2}, \quad\left(g_{11}(x)-g_{22}(x)\right) / x \text { are holomorphic. }
$$

Let $t$ be the $2 \times 2$ lower triangular complex matrices, $d \subset t$ be those with constant diagonals $\left(d_{11}=d_{22}\right)$. Let $T, D$ denote the corresponding groups. Then $H_{U}=D+x t+x^{2} \operatorname{gl}(2, \mathbb{C})+x^{3} \ldots$, and so one can truncate

$$
Q_{1}=\mathrm{Gl}(2, \mathbb{C})+x \operatorname{gl}(2, \mathbb{C}) / D+x t
$$


with the convention $x^{2}=0$. One has the commuting diagram of fibrations

$$
\mathrm{Gl}(2, \mathbb{C})+x \operatorname{gl}(2, \mathbb{C}) / T+x t \stackrel{Q_{1}}{\stackrel{\mathbb{C}^{*}}{\mathbb{C}}} \mathrm{Gl}(2, \mathbb{C}) / D
$$

Consider first the fibering (1) over (3). In (3), the equivalence with $\mathbb{P}_{1}$ is given by mapping $A \in \mathrm{Gl}(2, \mathbb{C})$ to the line $\left\langle A\left(\begin{array}{l}0 \\ 1\end{array}\right)\right\rangle$. Let $\mathcal{O}(-1)$ be the tautological bundle on $\mathbb{P}_{1}$. Then, $A+x B$ in (1) defines an element of $\mathcal{O}(2) \cong \operatorname{Hom}(\mathcal{O}(-1), \mathcal{O}(1))$ : to

$$
v \in \mathcal{O}(-1) \mid\left\langle A\left(\begin{array}{l}
0 \\
1
\end{array}\right)\right\rangle \text {, }
$$

one associates $\Pi_{A} \cdot B A^{-1}(v)$, where $\Pi_{A}$ is the projection $\mathbb{C}^{2} \rightarrow \mathbb{C}^{2} /\left\langle A\left(\begin{array}{l}0 \\ 1\end{array}\right)\right\rangle$; the fibration $(1) \rightarrow(3)$ is the bundle $\mathcal{O}(2)$ over $\mathbb{P}_{1}$.

Similarly, (2) over (3) can be written as

$$
\operatorname{Sl}(2, \mathbb{C}) /\left\{\left(\begin{array}{cc} 
\pm 1 & 0 \\
\alpha & \pm 1
\end{array}\right) \mid \alpha \in \mathbb{C}\right\} \rightarrow \operatorname{Sl}(2, \mathbb{C}) /\left\{\left(\begin{array}{cc}
\beta & 0 \\
\alpha & \beta^{-1}
\end{array}\right) \mid \alpha \in \mathbb{C}, \beta \in \mathbb{C}^{*}\right\}
$$

$\operatorname{Sl}(2, \mathbb{C}) /\left\{\left(\begin{array}{ll}1 & 0 \\ \alpha & 1\end{array}\right) \mid \alpha \in \mathbb{C}\right\}$ is just $\mathbb{C}^{2} \backslash\{0\}=(\mathcal{O}(-1))^{0}$, the tautological bundle minus the zero section; from this, one can see that (2) over (3) is the "square" of $(\mathcal{O}(-1))^{0}$, i.e. $(\mathcal{O}(-2))^{0}$. Using the diagram, one has:

Proposition 3.10. $Q_{1}$ is isomorphic to the fiber product $\mathcal{O}(2) \oplus(\mathcal{O}(-2))^{0}$ over $\mathbb{P}_{1}$. As $\mathcal{O}(-2)=T^{*} \mathbb{P}_{1}, Q_{1}$ is homotopy equivalent to the unit tangent bundle of the sphere, i.e. $\mathrm{SO}(3)$.

(iii) The Structure of $Q_{i}$. One could proceed similarly and define the space $Q_{i}$ as a quotient of the set of pairs $(f, g)$ of germs of maps from $\mathbb{C}, f$ into the versal deformation space $\mathbb{C}^{2 i-1}$ of $\mathcal{O}(i) \oplus \mathcal{O}(-i), g$ into $\mathrm{Gl}(2, \mathbb{C})$, by the natural equivalence relation. However, we lazily short-circuit this procedure, and note that $Q_{i}$ is nicely embedded in $\tilde{M}_{i}$ as $\Pi^{-1}(0)$, ensuring for example that $Q_{i}$ is a separated variety. Instead, we concentrate our attention on ${ }_{1} Q_{i}$, the open subset of $Q_{i}$ corresponding to jumps of type $(1,-1)$. Then, considering remark $(2.15)$, it is sufficient to replace $x$ by $x^{i}$ in (3.7), (3.8), and so obtain:

$$
{ }_{1} Q_{i}=\frac{\mathrm{Gl}(2, \mathbb{C})+x \mathrm{gl}(2, \mathbb{C})+\ldots+x^{2 i-1} \mathrm{gl}(2, \mathbb{C})}{D+x d+\ldots+x^{i-1} d+x^{i} t+\ldots+x^{2 i-1} t} .
$$

${ }_{1} Q_{i}$ is a bundle over ${ }_{1} Q_{(i-1)}$, with fiber $\mathbb{C}^{3}$. Remember also that (2.8) told us that any one parameter jump of multiplicity $k$ was the limit of jumps of multiplicity $k$, type $(1,-1)$, using the standard trivialisations. The same obviously remains true when one puts in the framings. Thus:

Proposition (3.11). ${ }_{1} Q_{i}$ is a $\mathbb{C}^{3 i-3}$ bundle over $Q_{1}$, and is homotopy equivalent to $\mathrm{SO}(3) ;{ }_{1} Q_{i}$ is open and dense in $Q_{i}$. 
The equidimensionality of the fibers $Q_{m_{1}} \times \ldots \times Q_{m_{r}}$ of $\Pi$ then follows, as this has dimension $\sum 3 m_{i}=3 n$.

Remarks. 1) One could try to use this procedure to construct the moduli space $R_{n}$ of all framed semi-stable 2-bundles $E$ on $\mathbb{P}_{2}$ with $c_{1}(E)=0, c_{2}(E)=n$. The problem is that there are $E$ 's which contain in their curves of jumping lines all the lines in our ruling; however, the variety $V_{n}$ of such bundles can be seen to be of codimension $\geqq 3$ in $R_{n}$ for $n \geqq 2$, and of codimension 2 for $n=1$, by considering the action of PGl(3) on $\mathbb{P}_{2}$ and the fact [5] that the generic curve of jumping lines is smooth. In fact, as all the lines in the ruling are jumps iff $(n+1)$ fixed lines are, a naive parameter count suggests $\operatorname{codim} V_{n}=(n+1)$. In any case, $R_{n} \backslash V_{n}$ fibers over $S^{n}\left(\mathbb{P}_{1}\right)=\mathbb{P}_{n}$.

2) One can see that the bundles in $M_{n}$ which are not stable form a subvariety of codimension $(n-1)$, as follows. $E$ is stable iff $H^{0}\left(\mathbb{P}_{2}, E\right)=0$. Any non-zero section of $E$ on $\mathbb{P}_{2}$ induces a non-zero covariant constant section along $C$. At the jumping lines, this section must lie in the $O(k), k>0$, summand. This means that the positive subbundles over the jumping lines must be "all lined up" at their intersection with $C$ with respect to the connection; there are thus $(n-1)$ constraints. One then checks, using the explicit description of $Q_{1}$, that for the case of $n$ distinct jumping lines, these constraints are sufficient. Multiple jumps may impose less constraints, but this is counterbalanced by the fact that they are themselves rarer.

One could use similar methods to determine which bundles on $\mathbb{P}_{2}$ have sections when twisted by $\mathcal{O}(j)$. One must, however, consider framings over the $j^{\text {th }}$ formal neighbourhood $C^{(j)}$ of $C . E(j)$, once lifted, has $(j+1)(j+2)$ independent sections over $C^{(j)}$; one checks that each jumping line imposes one condition, and so the $E$ 's with $H^{0}\left(\mathbb{P}_{2}, E(j)\right)$ non-zero have codimension at most $\max (n-(j+1)(j+2)+1,0)$.

3) One could use the method of (3.7), (3.8) to compute the different strata of $Q_{i}$ corresponding to jumps of type $(j,-j), j \leqq i$. This gets quite complicated rather quickly, though.

Let $\Delta$ be the discriminental variety in $S^{n}(C \backslash(\infty))=\mathbb{C}^{n}$; over $U$ simply connected in $\mathbb{C}^{n}-\Delta, M_{n}$ is just a product $U \times\left(Q_{1}\right)^{n}$; as branching around $\Delta$ interchanges the jumping lines, branching around $\Pi^{-1}(\Delta)$ interchanges the $Q_{1}$ 's. Over $\Delta$, the fiber becomes progressively more complicated as one considers jumps of higher multiplicity.

Finally, note that along the fibers of the map $\tilde{M}_{n} \rightarrow M_{n}$ (i.e., the same instanton with all its frames) the jumps remain the same; $M_{n}$ maps to $\mathbb{C}^{n}$. Furthermore, the fibers of $g$ are given by the diagonal action of $\mathrm{SU}(2)$ on the $Q_{m}$ 's of (3.5); one changes the frame simultaneously at all the jumps. Summing up:

Theorem (3.5a). There is a map

$$
\varrho: M_{n} \rightarrow S^{n}(C \backslash(\infty))=\mathbb{C}^{n},
$$

whose fiber at a point $\sum_{i=1}^{r} m_{i} p_{i}, p_{i} \in C \backslash(\infty)$, is:

$$
Q_{m_{1}} \times Q_{m_{2}} \times \ldots \times Q_{m_{r}} / \mathrm{SU}(2)
$$


where the quotient is taken with respect to the diagonal action on the $Q_{m}$ 's. All the fibers of $\varrho$ are of real dimension $6 n-3$.

\section{C) The Fundamental Group}

We have obtained a map $\tilde{M}_{n} \rightarrow \mathbb{C}^{n} \cong S^{n}(\mathbb{C})$. Let $\Delta$ be the discriminental variety in $\mathbb{C}^{n}$; if $U$ is simply connected and does not intersect $\Delta$, then $\Pi^{-1}(U)=U \times\left(Q_{1}\right)^{n}$. Let $\Delta_{0}$ be the smooth open dense subvariety of $\Delta$ consisting of those $n$-tuples in $S^{n}(\mathbb{C})$ having only two elements equal: the fibers of $\Pi$ over $\Delta_{0}$ are $Q_{2} \times\left(Q_{1}\right)^{(n-2)}$, and have an open dense subvariety ${ }_{1} Q_{2} \times\left(Q_{1}\right)^{(n-2)}$. Thus a $Q_{1} \times Q_{1}$ "degenerates" into a ${ }_{1} Q_{2}$; more precisely, let $U$ be a disk transversal to $\Delta_{0}$. Choosing appropriate coordinates, $a \in U$ corresponds to a polynomial of the form $\left(x^{2}-a\right)\left(x-p_{1}\right) \ldots\left(x-p_{n-2}\right),\left(p_{i}\right.$ distinct, $\left.\neq 0\right)$, whose roots are the $n$-tuple in $S^{n}(\mathbb{C})$. As in (3.8), (3.9), $\Pi^{-1}(a)$ is then the "variable" quotient (neglecting the extra $Q_{1}$ factors):

$$
\begin{gathered}
\{f: U \rightarrow \mathrm{Gl}(2, \mathbb{C})\} /\left\{f: U \rightarrow \mathrm{Gl}(2, \mathbb{C}) \mid f=f_{0}+\left(x^{2}-a\right) f_{1}+\left(x^{2}-a\right)^{2} f_{2},\right. \\
\left.f_{0}: U \rightarrow D, f_{1}: U \rightarrow t, f_{2}: U \rightarrow \operatorname{gl}(2, \mathbb{C})\right\} .
\end{gathered}
$$

$\Pi^{-1}(a)$ is then $Q_{1} \times Q_{1}$ for $a \neq 0$, by evaluation at $\pm \sqrt{a}$, and is ${ }_{1} Q_{2}$ for $a=0$.

We thus have a good description of $\tilde{M}_{n}^{1}$, the space of bundles in $\Pi^{-1}\left(\left(\mathbb{C}^{n} \backslash \Delta\right) \cup \Delta_{0}\right)$ with only jumps of type $(1,-1)$ in the ruling. $\tilde{M}_{n} \backslash \tilde{M}_{n}^{1}$ is of complex codimension 2 (real codimension 4$)$ in $\tilde{M}_{n}$; therefore, $\pi_{1}\left(\tilde{M}_{n}^{1}\right)=\pi_{1}\left(\tilde{M}_{n}\right)$, and $\pi_{2}\left(\tilde{M}_{n}^{1}\right)=\pi_{2}\left(\tilde{M}_{n}\right)$. We use this to prove:

Theorem (3.13). $\pi_{1}\left(\tilde{M}_{n}\right)=\mathbb{Z}_{2}$.

Proof. First, note that loops $L$ and their homotopies can be supposed differentiable; transversality arguments then imply that $L$ may be taken so that $L \cap \Pi^{-1}\left(\Delta_{0}\right)=0$, and homotopies of $L$ may chosen to intersect $\Pi^{-1}\left(\Delta_{0}\right)$ in discrete points.

Suppose then that one has a loop $L$ in $\tilde{M}_{n}^{1}$ projecting to $\mathbb{C}^{n} \backslash \Delta$. Over $\mathbb{C}^{n} \backslash \Delta, \tilde{M}_{n}^{1}$ is locally just a product, and so there one can lift a homotopy in $\mathbb{C}^{n} \backslash \Delta$ to $\tilde{M}_{n}^{1}$. Furthermore, in a neighbourhood of $\Delta_{0}$, using (3.12), a segment $I$ of $L$ can be lifted to a map $I \times U \rightarrow \mathrm{Gl}(2, \mathbb{C})$ and so be "pushed" in a natural way through $\Pi^{-1}\left(\Delta_{0}\right)$ :
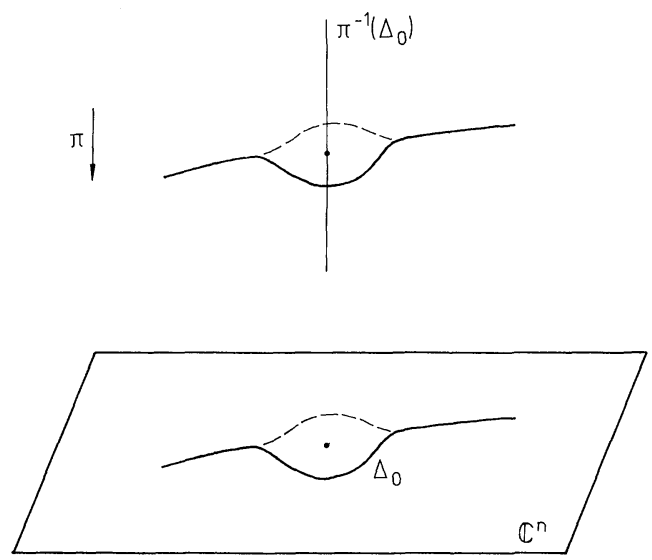
One can therefore lift a contraction of $\Pi(L)$ to $\tilde{M}_{n}^{1} ; L$ can thus be pushed onto a fiber $\left(Q_{1}\right)^{n} . \pi_{1}\left(\tilde{M}_{n}\right)$ is then a quotient of $\pi_{1}\left(\left(Q_{1}\right)^{n}\right)=\left(\mathbb{Z}_{2}\right)^{n}$ and $H_{1}\left(\tilde{M}_{n}, \mathbb{Z}\right)=\pi_{1}\left(\tilde{M}_{n}\right)$. Now remark that branching around $\Delta$ interchanges roots, and so branching around $\Pi^{-1}(\Delta)$ interchanges the $Q_{1}$ 's. The loop $(1,0, \ldots, 0)$ in $\left(\mathbb{Z}_{2}\right)^{n} \cong \pi_{1}\left(\left(Q_{1}\right)^{n}\right)$ is therefore homotopic in $\tilde{M}_{n}$ to the loop $(0,0, \ldots, 0,1,0, \ldots, 0)$. Furthermore, $(1,0, \ldots, 0)$ is not homotopic to zero, as any homotopy away from $\Pi^{-1}\left(\Delta_{0}\right)$ cannot contract it, and pushing it through $\Pi^{-1}\left(\Delta_{0}\right)$ just sends it to a $(0,0, \ldots, 1,0, \ldots, 0)$. Therefore $\pi_{1}\left(\tilde{M}_{n}\right)=\mathbb{Z}_{2}$.

Theorem 3.15. Let $M_{n}$ be the moduli space of SU(2) instantons of charge $n$. Then $\pi_{1}\left(M_{n}\right)=0$ if $n$ is odd, $\mathbb{Z}_{2}$ if $n$ is even.

Proof. The only automorphisms of instanton bundles over $\mathbb{P}_{3}$ are homotheties ([13], p. 172), and so $\tilde{M}_{n}$ fibers over $M_{n}$ with fiber $\mathrm{SU}(2) / \pm 1=\mathrm{SO}(3)$. The natural SU(2) action on $\tilde{M}_{n}$ is given by the simultaneous standard left action of SU(2) C Gl $(2, \mathbb{C})$ on the $Q_{i}^{\text {'s }}$ of $(3.5)$. In particular, over $\Pi^{-1}\left(\mathbb{C}^{n} \backslash \Delta\right)$, the fiber of $\tilde{M}_{n} \rightarrow M_{n}$ is just the orbit of the diagonal left action of $\mathrm{SU}(2)$ on $n$ copies of (3.9). $\pi_{1}(\mathrm{SO}(3))$ thus maps to the diagonal $\mathbb{Z}_{2}$ in $\left(\mathbb{Z}_{2}\right)^{n}=\pi_{1}\left(\left(Q_{1}\right)^{n}\right)$ and so, from the proof of (3.13), onto zero if $n$ is even, $\pi_{1}\left(\tilde{M}_{n}\right)$ if $n$ is odd, once one maps to $\pi_{1}\left(\tilde{M}_{n}\right)$. The result then follows from the exact homotopy sequence

$$
\ldots \rightarrow \pi_{1}(\mathrm{SO}(3)) \rightarrow \pi_{1}\left(\tilde{M}_{n}\right) \rightarrow \pi_{1}\left(M_{n}\right) \rightarrow 0 .
$$

Remarks. 1) As $\pi_{2}\left(Q_{1}\right)=\pi_{2}\left({ }_{1} Q_{2}\right)=0$ it is natural to conjecture, similarily to (3.13), that $\pi_{2}\left(\tilde{M}_{n}\right)=0$. This then implies that $\pi_{2}\left(M_{n}\right)=0$ if $n$ is odd, $\mathbb{Z}_{2}$ if $n$ is even.

2) (3.15) concurs with what is known for $n=1,2 . M_{1}$ is the 5-ball (Atiyah [1]) and $M_{2}$ is not simply connected (Hartshorne [11]).

3) Le Potier [14] gives a computation of $\pi_{1}$ of the moduli space of stable 2-bundles over $\mathbb{P}_{2}$.

\section{D) Link With Monads}

We now show how our geometric picture links up with the more algebraic one of monads. For simplicity, we will treat only the case of $n$ distinct jumping lines along the divisor $C$; our aim is to give ourselves the geometric information corresponding to our picture, and to extract from it a monad.

First, recall that a semi-stable rank 2 bundle $E$ with $c_{1}(E)=0, c_{2}(E)=n$ can be represented by a monad $[13,6]$

$$
\mathbb{C}^{n} \stackrel{H_{p}}{\longrightarrow} \mathbb{C}^{2 n+2} \stackrel{K_{p}}{\longrightarrow} \mathbb{C}^{n}
$$

where, if $p=(X, Y, W)$ in homogeneous coordinates, $H_{p}=H_{x} X+H_{y} Y+H_{w} W$, $K_{p}=K_{x} X+K_{y} Y+K_{w} W$, and one has, for all $p, K_{p} H_{p}=0 . E$, at $p$, is given by $E_{p}=\operatorname{ker} K_{p} / \operatorname{Im} H_{p}$. The bundle is trivial along a line $\overline{p_{1} p_{2}}$ iff $K_{p_{1}} H_{p_{2}}=-K_{p_{2}} H_{p_{1}}$ is an isomorphism; $H^{0}\left(\overline{p_{1} p_{2}}, E\right)=\operatorname{ker} K_{p_{1}} \cap \operatorname{ker} K_{p_{2}}$; evaluation of a section at $p_{2}$ is just the natural map to $\operatorname{ker}\left(K_{p_{2}}\right) / \operatorname{Im}\left(H_{p_{2}}\right)[13,6]$.

Suppose that the bundle is trivial along $w=0$; then one can choose bases so that, decomposing the $H$ 's into blocks of $n \times n, n \times n, 2 \times n$, and the $K$ 's into blocks of $n \times n, n \times n, n \times 2$,

$$
\begin{gathered}
H_{x}=\left(\begin{array}{c}
\mathrm{Id} \\
0 \\
0
\end{array}\right), \quad H_{y}=\left(\begin{array}{c}
0 \\
\mathrm{Id} \\
0
\end{array}\right), \quad H_{w}=\left(\begin{array}{c}
\alpha_{1} \\
\alpha_{2} \\
a
\end{array}\right), \\
K_{x}=(0, \mathrm{Id}, 0), \quad K_{y}=(-\mathrm{Id}, 0,0), \quad K_{w}=\left(-\alpha_{2}, \alpha_{1}, b\right),
\end{gathered}
$$


with

$$
\left[\alpha_{1}, \alpha_{2}\right]+b a=0
$$

one has an action of $\mathrm{Gl}(n, \mathbb{C})$ on these, preserving $E$ and a natural framing of $E$ along $W=0$ :

$$
\alpha_{i} \rightarrow p \alpha_{i} p^{-1}, \quad a \rightarrow a p^{-1}, \quad b \rightarrow p b, \quad p \in \mathrm{Gl}(n, \mathbb{C}) .
$$

The line $W=0$ will correspond to our $l_{\infty}$ used previously, and we choose our point on $l_{\infty}$ to be $(0,1,0)$. We are therefore interested in the pencil of lines through $(0,1,0)$; if one blows up $(0,1,0)$ to obtain $C$, the coordinates used previously are given here by $x=X / W, z=W / Y ; C$ is $(z=0)$ and the lines through $C$ are ( $x=$ const). Thus, in accordance with our description of the moduli space, our geometric data will be the choice of $n$ different jumping lines $x=x_{i}$, and, in a neighbourhood of each $x_{i}$, a map into $\mathrm{Gl}(2, \mathbb{C}), g_{i}(x)=g_{i}+\left(x-x_{i}\right) \tilde{g}_{i}+\ldots$. It is from these that we must obtain a monad.

The line $\left(x=x_{0}\right)$ is also $\overline{p_{1} p_{2}}, p_{1}=(0,1,0), p_{2}=\left(x_{0}, 0,1\right)$; thus $K_{p_{1}} H_{p_{2}}$ $=\left(\alpha_{1}+x_{0} \cdot \mathrm{Id}\right)$. This is singular if $x_{0}=x_{i}$, and so, using our $\operatorname{Gl}(n, \mathbb{C})$ freedom, one can set:

$$
\alpha_{1}=-\operatorname{diag}\left(x_{1}, \ldots, x_{n}\right)
$$

one still has a $\left(\mathbb{C}^{*}\right)^{n}$ action fixing this form. From $\left(\left[\alpha_{1}, \alpha_{2}\right]+\right.$ ba $)=0$,

$$
\left(\alpha_{2}\right)_{i j}\left(x_{i}-x_{j}\right)=(b a)_{i j} \text {. }
$$

Suppose that $E$ is lifted to $\widetilde{\mathbb{P}}_{2}$, and that the result is again called $E$. We investigate maps $\left.\left.E\right|_{(x, 0)} \rightarrow E\right|_{(x, z)}$. Set $(x, z)=q$; our first map is the "connection" map:

$$
F:\left.E\right|_{(x, 0)}=\left.\left.E\right|_{p_{1}} \rightarrow H^{0}\left(\overline{p_{1} q}, E\right) \rightarrow E\right|_{q},
$$

defined over the non-jumping lines $\left(x \neq x_{i}\right)$. As

$F$ is just: $\quad \operatorname{ker} K_{p_{1}} \cap \operatorname{ker} K_{q}=\left\{\left(0,-\left(\alpha_{1}+x\right)^{-1} b w, w\right)^{T}, w \in \mathbb{C}^{2}\right\}$,

$$
w \rightarrow\left(0,-\left(\alpha_{1}+x\right)^{-1} b w, w\right) \text {, considered } \bmod H_{\left(x, z^{-1}, 1\right)}\left(\mathbb{C}^{n}\right)=\left(\alpha_{2}+z^{-1}\right) \cdot \mathbb{C}^{n} .
$$

This map is singular at the jumping lines $x=x_{i}$; however, there is also the map $F_{T}$ over $z \neq \infty$ induced by any homomorphic trivialisation $T$ of $E$ over $z \neq \infty$ : one associates to $\left.v \in E\right|_{(x, 0)}$ the $\left.w \in E\right|_{(x, z)}$ with the same coordinates. This is nonsingular at $x=x_{i}$, and we look at it near $x=x_{i}$. In the terminology of (3.7), the passage in coordinates from the "connection," (or "sections") trivialisation to a holomorphic trivialisation over $z \neq \infty$ is given by $A^{-1}(x, z) g_{i}(x)^{-1}$. Therefore,

$$
F_{T}=g_{i}(x) A(x, z) F A^{-1}(x, 0) g_{i}^{-1}(x) \text {. }
$$

As $A(x, 0)=\mathrm{Id}$, setting $v=g^{-1}(x) w$, one is considering, for the image of $F_{T}$,

$$
\left(0,-\left(\alpha_{1}+x\right)^{-1} b g_{i}(x) A(x, z) v, g_{i}(x) A(x, z) v\right)^{T},
$$

$\bmod \operatorname{Im}\left(H_{\left(x, z^{-1}, 1\right)}\right)$. At $x=x_{i}$, (3.19) has a polar part, which must lie in $\operatorname{Im}\left(H_{\left(x, z^{-1}, 1\right)}\right)$, as $F_{T}$ is continuous. One then checks that this happens, if and only if, up to the remaining $\left(\mathbb{C}^{*}\right)^{n}$ action,

$$
a_{i}=g_{i} \cdot(0,1)^{T}, \quad b_{i}=(-1,0) \cdot\left(g_{i}\right)^{-1}, \quad\left(\alpha_{2}\right)_{i i}=(1,0) \cdot\left(g_{i}\right)^{-1} \tilde{g}_{1}(0,1)^{T},
$$

which, along with (3.16), and (3.17), gives us our monad. 


\section{References}

1. Atiyah, M.F.: The geometry of Yang-Mills fields. Accademia Nazionale dei Lincei, Scuola Normale Superiore, Pisa 1979

2. Atiyah, M.F.: Instantons in two and four dimensions. Commun. Math. Phys. 93, 437-451 (1984)

3. Atiyah, M.F., Drinfeld, V.G., Hitchin, N.J., Manin, Yu.I.: Construction of instantons. Phys. Lett. 65 A, 185-187 (1978)

4. Barth, W.: Some properties of stable rank-2 vector bundles on $\mathbb{P}_{n}$. Math. Ann. 226, 125-150 (1977)

5. Barth, W.: Moduli of vector bundles on the projective plane. Invent. Math. 42, 63-91 (1977)

6. Donaldson, S.K.: Instantons and geometric invariant theory. Commun. Math. Phys. 93, 453-461 (1984)

7. Grauert, H., Mülich, G.: Vektorbündel vom Rang 2 über dem n-dimensionalem komplex projektiven Raum. Manuscr. Math. 16, 75-100 (1975)

8. Grothendieck, A.: Eléments de géométrie algébrique. III. Publ. Math. IHES

9. Grothendieck, A.: Sur la classification des fibres holomorphes sur la sphère de Riemann. Am. J. Math. 79, 121-138 (1957)

10. Hartshorne, R.: Algebraic geometry. Berlin, Heidelberg, New York: Springer GTM (1978)

11. Hartshorne, R.: Stable vector bundles and instantons. Commun. Math. Phys. 59, 1-15 (1978)

12. Horrocks, G.: Vector bundles on the punctured spectrum of a local ring. Proc. Lond. Math. Soc. (3) 14, 689-713 (1964)

13. Okonek, C., Schneider, M., Spindler, H.: Vector bundles on complex projective spaces. Prog. Math. 3. Boston: Birkhäuser 1980

14. Le Potier, J.: Fibres stables de rang 2 sur $\mathbb{P}_{2}(\mathbb{C})$. Math. Ann. 241, 217-256 (1979)

Communicated by A. Jaffe

Received November 26, 1985; in revised form February 10, 1986 